\title{
The $J C l$ starts two new series: Science in Medicine and Science and Society
}

In this issue, the JCI inaugurates a new series on Science in Medicine. With this series and another on Science and Society, the journal seeks to expand its role as a voice for important issues that impact the academic health care community. The Science in Medicine series is designed to help bridge the gap between scientific discoveries made at the bench and the practice of medicine. The first article in the Science in Medicine series examines how advances in the understanding of the molecular pathophysiology of asthma have formed the basis for novel and effective therapeutics that are used daily in patient care (1). The Science and Society series looks to explore the meaning behind society's many ways (e.g., governmental policy, economics, ethics, religion, and politics) of molding the course of biomedical education and research. The first articles in the Science and Society series addressed the complexities that intellectual property rights have introduced into the conduct of biomedical research (2) and shortcomings in the infrastructure of science education in the United States (3). Future articles will focus on the decline of the academic mission in U.S. health centers, the future of biomedical research publishing, and the impact of politics on scientific research.

Last spring the JCI conducted a readership survey to help guide the new Editorial Board in plotting a course for the journal over the next five years. Interestingly, of the 551 respondents, $96 \%$ of whom indicated that they are involved in biomedical research, over $67 \%$ reported that they also participate in patient care and teaching. This response supports the editors' desire to enhance the scope of the JCI and make it an important journal for practicing academic physicians to read on a regular basis. It is our hope that the Science in Medicine series will become a valuable asset to the teaching physician on rounds and to medical students and house staff. As one anonymous respondent wrote, "It would be good to make [the JCI] more appealing to clinicians who do not do basic research but are interested in basic research." Ultimately, better-informed physicians are better physicians for their patients.

The editors have asked the authors of Science in Medicine series articles to present historical perspectives of common diseases in which they are recognized experts as well as comprehensive reviews of the contribution of
"New Biology" to understanding mechanisms, diagnostics, and therapeutics of these diseases. While the emphasis will be on the present-day practice of medicine, insights into future directions for new therapeutics will also be included. The goal is to write the kind of paper that attendings (clinicians) will want to bring on rounds with them to help teach house staff and medical students.

In the JCI survey, $67 \%$ of respondents indicated that they still prefer the print version of the JCI, even though it is available, for free, on-line. Indeed, the JCI is the only major peer-reviewed biomedical research journal that is available barrier-free on-line. This includes all of the front matter of the journal as well as all scientific articles. However, it is likely that the front matter is read more completely by subscribers to the print version of the journal, and the editors are investing considerable energy toward increasing the value of this front matter to the readership.

These new efforts are in line with the editors' vision for the JCI as the premier journal for peerreviewed biomedical research and commentary. In a sense, these changes do not represent new directions for the JCI, which, for the past three quarters of a century, has been a leading platform for the exposition of biomedical research. Rather, the new initiatives look to fulfill a promise that can best be met by a journal with sufficient history and of substantial quality to serve a community of increasing diversity in a time when informational overload threatens to become a new diagnosis. All of these efforts will depend on the continued good will and incredible energy provided by the contributors, reviewers, editors, and staff who labor with affection to produce a good read that provokes thought and contributes meaningfully to the body of scientific knowledge.

\section{Andrew R. Marks}

Editor-in-Chief

Columbia University College of Physicians and Surgeons

1. Elias, J.A., et al. 2003. New insights into the pathogenesis of asthma. J. Clin. Invest. 111:291-297. doi:10.1172/JCI200317748.

2. Dove, A. 2002. When science rides the MTA. J. Clin. Invest. 110:425-427. doi:10.1172/JCI200216546.

3. Dove, A. 2002. It takes a village to raise an idiot: fixing US science education. J. Clin. Invest. 110:1057-1060. doi:10.1172/JCI200216959. 